\title{
Forecasting the Cumulative Confirmed Cases with the FGM and Fractional-Order Buffer Operator in Different Stages of COVID-19
}

\author{
Yanhui Chen (D), Minglei Zhang, Kai Lisa Lo $(\mathbb{D}$, and Jackson Jinhong Mi \\ School of Economics and Management, Shanghai Maritime University, Shanghai 201306, China \\ Correspondence should be addressed to Yanhui Chen; chenyh@shmtu.edu.cn and Kai Lisa Lo; kllo@shmtu.edu.cn
}

Received 16 June 2021; Accepted 30 September 2021; Published 26 October 2021

Academic Editor: Lifeng Wu

Copyright (C) 2021 Yanhui Chen et al. This is an open access article distributed under the Creative Commons Attribution License, which permits unrestricted use, distribution, and reproduction in any medium, provided the original work is properly cited.

\begin{abstract}
This study proposes to use the fractional-order accumulation grey model (FGM) combined with the fractional-order buffer operator to predict the cumulative confirmed cases in different stages of COVID-19. In the early stages of COVID-19 outbreak, when the cumulative confirmed cases increased rapidly, we used the strengthening buffer operator in the prediction process. After the government's prevention measures started to take effect, the growth rate of cumulative confirmed cases slows down. Therefore, the weakening buffer operator is applied in the prediction process. The fractional order of the buffer operator is derived from the historical data, which are more relevant. The empirical analysis of seven countries' data shows that the FGM with the fractionalorder buffer operator achieves the best results for most cases. The fractional-order buffer operator improves the prediction accuracy of the FGM in this study. Our study also suggests a practical way for predicting the trend of epidemic diseases.
\end{abstract}

\section{Introduction}

Fractional-order system is a more extensive complex system than the integer-order system, which has attracted extensive attention from academia and industry in recent years. The fractional-order system is more flexible, which realizes the precise modeling of complex systems; compared with the traditional integer-order controller, additionally, the fractional-order controller has advantages in robustness and design freedom. Many valuable achievements have been made in numerical calculation [1], system modeling [2], stability analysis [3], and integrated control [4] under the concept of fractional-order systems.

In the predictions of complex systems, an inevitable problem is how to forecast the system in the early stage or with limited existing data. Under the condition of less data environment, the grey system theory makes full use of the known "minimum information" and gives priority to extract the more valuable "new information," realizing the modeling of the whole system. It can be used to predict the changing trend of the system in the future $[5,6]$. One of the most useful methods under this theory is $\operatorname{GM}(1,1)$, which is suitable to predict the data with an exponential growing trend. Researchers used GM $(1,1)$ to solve the prediction problems in consumption demand, pollutant emission, and energy utilization [7-10]. The advantage of GM $(1,1)$ is prediction with limited data; however, it is more suitable for the data with an exponential growing trend. In order to improve the prediction accuracy of limited data with nonexponential growth trends, many scholars derived more models from traditional GM $(1,1)$, such as the grey Verhulst model $[11,12]$, grey Bernoulli model $[13,14]$, and fractionalorder accumulation grey model (FGM) [15]. Since the fractional-order accumulation grey model solves the problem that the GM $(1,1)$ model cannot be used to predict the data that do not follow the grey exponential law completely accurately, it has attracted researchers' attention widely, such as Liu et al. [16], Zeng [17], Wu et al. [18], and Liu et al. [19].

The predictions of complex systems also face another problem that is how to predict the future trend of the system changes accordingly when the development of the system is disturbed. The problem in forecasting at this time does not lie in the pros and cons of the model but in the fact that the information provided by the system cannot accurately reflect the future trend. It is difficult to use the information directly 
to make an accurate prediction. In order to deal with this problem in system prediction, Liu [20] proposed the concept of buffer operator in the grey system prediction models. The operator which slows down the growth rate of the sequence is called the weakening buffer operator (WBO). The operator which speeds up the growth rate of the sequence is called the strengthening buffer operator (SBO). Many scholars have proposed a variety of buffer operators based on different data to accurately predict the system with disturbance [21-23]. Buffer operator of grey system theory combines the quantitative and empirical prediction (qualitative prediction) because in these literature studies, the researchers used their experiences to analyze the future trend qualitatively and proposed a new form of buffer operators. This means that the buffer operators they built may have the problem of poor versatility. Recently, Wu et al. [24] generalized the expression of weakening and strengthening buffer operators using the fractional order, which provides us a new choice to generate different buffer operators based on different fractional orders.

Infectious diseases have always been a major public health concern. Historically, the large-scale outbreaks of infectious diseases have brought great trauma to human society. Recently, relying on modern science and technology, we have found the causes of many infectious diseases, summarized the control methods, and found the effective treatment methods of most infectious diseases. Yet, the pathogens of infectious diseases are constantly mutating and evolving, and they have struggled with human beings for a long time. The outbreak of COVID-19 is just the case. This disease is caused by SARs-CoV-2 which is a variant coronavirus. It has similar symptoms to cold and flu if the symptoms are mild, but this new coronavirus has stronger concealment and infectivity. Patients often develop symptoms long after they are infected with the virus. In addition to droplet transmission, the virus can also be transmitted through contact, aerosol transmission. Patients in the incubation period also have strong infectious power. Besides attacking our lungs, the virus can also damage other organs. Severe patients often lose their lives due to multiple organ failure, but at the very beginning, when COVID-19 broke out in Wuhan, China, the doctors knew nothing about this virus. A lot of people were infected. Doctors and scientists had to gradually deepen their understanding of the virus during the treatment of patients. We now know the nature of the coronavirus, and face masks can protect us from infection. Keeping social distance and home quarantine can slow down the spread of the virus. Although some governments did not take effective epidemic control measures in the very beginning, after the number of infected people in their own countries soared, these countries began to adopt strict control measures.

Ever since the outbreak of COVID-19, the prediction of the number of confirmed cases has attracted the attention of researchers. Generally speaking, three types of methods are mainly adopted in the literature. The first type is the time series regression model, in which ARMA/ARIMA is a commonly employed model. Perone [25] constructed the ARIMA model to predict the number of confirmed cases at the national and regional levels in Italy. Sharma et al. [26] and Ilie et al. [27] also used this method for prediction. The second uses machine learning. Tameng et al. [28] predicted the confirmed cases and deaths in India, the United States, France, and the United Kingdom through the curve fitting technique based on the artificial neural network (ANN). Hazarika and Gupta [29] predicted the COVID-19 spread in five worst-hit countries by combining the random vector function chain network (RVFL) method with wavelet transform. Chimmula and Zhang [30] used the LSTM neural network to predict the confirmed cases in Canada. The third type is the epidemic dynamic model-SIR (susceptible-infection-recovered). Based on this model, the researchers extended their model according to the particularity of COVID-19. Khajanchi and Sarkar constructed a new SAIUQR model to predict the numbers of daily and cumulative confirmed cases in four states in India [31]. Cooper et al. augmented the classic SIR model with the ability to accommodate surges in the number of susceptible individuals to predict the community confirmed cases in five countries [32].

Grey system methods have their advantage of predicting the COVID-19 epidemic as it is shown that they are effective for predicting exponential growth data. Liu et al. predicted the number of early confirmed cases in China by the fractional grey model [19]. However, with the development of the epidemic situation, it has been proved that the epidemic control measures, such as lockdown/quarantine, have effectively slowed down the rise in the number of infected people $[33,34]$. Exponential growth is no longer appropriate to describe the increase in COVID-19's cumulative number of confirmed cases. Besides, according to the stage of the epidemic, it is necessary to choose the forecasting methods. This paper proposes to use different fractional-order buffer operators integrated with the FGM to forecast the trend of COVID-19.

In this paper, we name the order of the generalized strengthening and weakening buffer operator as the buffer level. By using the generalized strengthening and weakening buffer operator, the researchers need not build a new buffer operator in their research studies; all they need is to decide an order according to their experiences or generate an order based on similar cases. In the empirical analysis, this paper uses the prediction of COVID-19 cumulative confirmed cases in the early stage and after the governments' epidemic control measures were in effect to demonstrate the use of the fractional-order buffer operator in the grey system model. Our main contribution is threefold. Firstly, this study introduces different kinds of buffer operators in different stages of the epidemic. Secondly, different from previous studies in which the fractional order of the buffer operator is determined based on the experts' experiences, this paper uses the historical data to estimate the fractional order of the buffer operator and proposes an applicable prediction process (see the last paragraphs in Sections 3.1 and 3.2). Thirdly, this paper takes into account both government control measures and public responses in predicting cumulative confirmed cases. 
The following of the paper includes three sections. Section 2 introduces the method, Section 3 demonstrates the empirical results, and Section 4 concludes the paper briefly.

\section{Methodology}

2.1. Fractional-Order Accumulation Grey Model. GM $(1,1)$ is the most classical and basic model in grey system theory. Driven by practice, academic research on GM $(1,1)$ has been very active over the past three decades. Scholars derive many models from traditional GM $(1,1)$ to improve the forecast precision and apply them to more new fields. Fractionalorder accumulation grey model (FGM) is one of these derived models. It uses the fractional accumulate order to make the historical data in the weighted form. The order is very important in the FGM. The larger the order is, the larger the weight of old data is. Increasing the order can increase the weight of old data, which puts less emphasis on the newer data, and vice versa. As a result, it can extract the pivotal information of the historical data and make accurate prediction. A standard FGM $(1,1)$ is established as follows.

Definition 1. It is assumed that the original sequence is

$$
X^{(0)}=\left(x^{(0)}(1), x^{(0)}(2), \ldots, x^{(0)}(n)\right) .
$$

$X^{(0)}$ is a nonnegative sequence, while the $r$-AGO $(0<r<1)$ sequence of $X^{(0)}$ is

$$
X^{(r)}=\left(x^{(r)}(1), x^{(r)}(2), \ldots, x^{(r)}(n)\right),
$$

where

$$
x^{(r)}(k)=\sum_{i=1}^{k} C_{k-i+r-1}^{k-i} x^{(0)}(i), \quad k=1,2, \ldots, n .
$$

According to the definition of the gamma function, $X^{(r)}$ can be rewritten in the form of gamma function as follows:

$$
x^{(r)}(k)=\sum_{i=1}^{k} \frac{\Gamma(k-i+r)}{\Gamma(k-i+1) \cdot \Gamma(r)} x^{(0)}(i), \quad k=1,2, \ldots, n .
$$

$Z^{(r)}$ represents the generated mean sequence of consecutive neighbors of $X^{(r)}$.

$$
Z^{(r)}=\left(z^{(r)}(1), z^{(r)}(2), \ldots, z^{(r)}(n)\right),
$$

where

$$
Z^{(r)}(k)=\frac{\left(x^{(r)}(k)+x^{(r)}(k+1)\right)}{2}, \quad k=1,2, \ldots, n-1 .
$$

Definition 2. If $X^{(0)}, X^{(r)}$, and $Z^{(r)}$ are described as Definition 1 , the following formula is obtained:

$$
x^{(r)}(k)-x^{(r)}(k-1)+a z^{(r)}(k)=u .
$$

This formula is the original form of $\operatorname{FGM}(1,1)$, and

$$
\frac{\mathrm{d} x^{(r)}}{\mathrm{d} t}+a x^{(r)}=u
$$

is the whitening equation of FGM $(1,1)$.

Theorem 1. If $X^{(0)}$ and $X^{(r)}$ are described as Definition 1, the parameter vector $\widehat{a}=[a, u] T$ can be estimated by the least square method as follows:

$$
\widehat{a}=\left(B^{T} B\right)^{-1} B^{T} Y,
$$

where

$$
B=\left[\begin{array}{cc}
-\frac{x^{(r)}(1)+x^{(r)}(2)}{2} & 1 \\
-\frac{x^{(r)}(2)+x^{(r)}(3)}{2} & 1 \\
\vdots & \vdots \\
-\frac{x^{(r)}(n-1)+x^{(r)}(n)}{2} & 1
\end{array}\right] \text {, }
$$

$$
Y=\left[\begin{array}{c}
x^{(r)}(2)-x^{(r)}(1) \\
x^{(r)}(3)-x^{(r)}(2) \\
\vdots \\
x^{(r)}(n)-x^{(r)}(n-1)
\end{array}\right] .
$$

The solution to the whitening equation of $\operatorname{FGM}(1,1)$ is shown as follows:

$$
\widehat{x}^{(r)}(k)=\left(x^{(0)}(1)-\frac{u}{a}\right) e^{-a(k-1)}+\frac{u}{a} .
$$

By substituting the parameter vector $\widehat{a}=[a, u] T$ into formula (11), the fitting value sequence $\widehat{X}^{(r)}$ can be obtained. The fitting value sequence $\widehat{X}^{(0)}$ can be calculated by the $r$ order inverse accumulation.

$$
\begin{aligned}
\widehat{X}^{(1)}= & \left(\hat{x}^{(1)}(1), \hat{x}^{(1)}(2), \ldots, \hat{x}^{(1)}(n+1), \ldots\right) \\
= & \left(\hat{x}^{(r)(1-r)}(1), \hat{x}^{(r)(1-r)}(2), \ldots, \hat{x}^{(r)(1-r)}(n+1), \ldots\right), \\
& \hat{x}^{(0)}(k)=\hat{x}^{(1)}(k+1)-\hat{x}^{(1)}(k), \\
\widehat{X}^{(0)}= & \left(\hat{x}^{(0)}(1), \hat{x}^{(0)}(2), \ldots, \hat{x}^{(0)}(n+1), \ldots\right) .
\end{aligned}
$$

2.2. Buffer Operator with the Fractional Order. When a system is affected by a shock, the existing data cannot reflect the law of the system truthfully. In this case, buffer operator of the grey system was proposed to revise the future trend. It combines quantitative and judgmental forecast (qualitative analysis). Recently, several buffer operators have been proposed and used in many fields successfully [35-37]. The basic idea of using the buffer operator in the prediction 


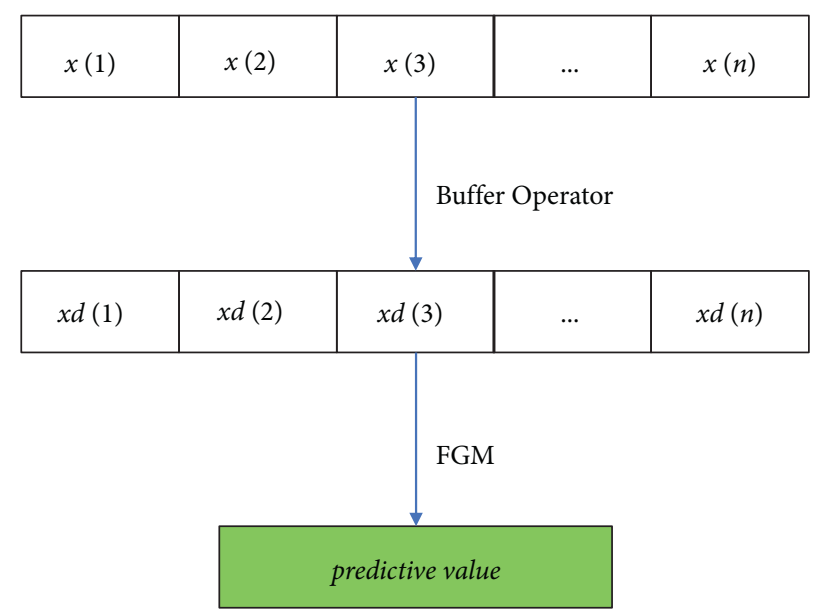

Figure 1: The basic idea of prediction with the buffer operator.

models is the trend of the original data which is modified by the buffer operator. Through the strengthening buffer effect, the original data turn into a new dataset, which shows more rapid growth. Through the weakening buffer effect, the original data turn into a new dataset with slower growth. As the prediction should be based on the historical data, it is necessary to modify the historical data which are more convenient for prediction modeling. Buffer operator is used to adjust the historical data to make it consistent with the future possible development trend which will show more rapid or slower growth according to the experience and expert's judgement. This transform would be conducive to accurate prediction. Wu et al. [24] extended the traditional integer-order buffer operator to the fractional-order one.

Definition 3. Assume that the raw data sequence is $X=x(1), x(2), \ldots, x(n)$.

$$
D=\left[\begin{array}{cccc}
\frac{1}{n} & 0 & \cdots & 0 \\
\frac{1}{n} & \frac{1}{n-1} & \cdots & 0 \\
\vdots & \vdots & \ddots & \vdots \\
\frac{1}{n} & \frac{1}{n-1} & \cdots & 1
\end{array}\right] .
$$

Then, $p / q$ order of the weakening buffer operator is

$$
X D^{p / q}=X\left[\begin{array}{cccc}
\frac{1}{n} & 0 & \cdots & 0 \\
\frac{1}{n} & \frac{1}{n-1} & \cdots & 0 \\
\vdots & \vdots & \ddots & \vdots \\
\frac{1}{n} & \frac{1}{n-1} & \cdots & 1
\end{array}\right]^{p / q}
$$

Definition 4. For original data $X=x(1), x(2), \ldots, x(n)$, the $p / q$ order of the strengthening buffer operator is

$$
\begin{aligned}
& X D^{-(p / q)}=X\left[\begin{array}{cccc}
\frac{1}{n} & 0 & \cdots & 0 \\
\frac{1}{n} & \frac{1}{n-1} & \cdots & 0 \\
\vdots & \vdots & \ddots & \vdots \\
\frac{1}{n} & \frac{1}{n-1} & \cdots & 1
\end{array}\right]^{-(p / q)} \\
& =X\left[\begin{array}{ccccc}
n & 0 & 0 & \cdots & 0 \\
-(n-1) & n-1 & 0 & \cdots & 0 \\
0 & -(n-2) & n-2 & \cdots & 0 \\
\vdots & \vdots & \vdots & \ddots & \vdots \\
0 & 0 & 0 & \cdots & 1
\end{array}\right]^{p / q}
\end{aligned}
$$

The value of $p / q$ is noted as the buffer level in the following sections. The prediction procedure is demonstrated briefly in Figure 1.

\section{Empirical Analysis}

3.1. The Prediction of COVID-19 Confirmed Cases in the Early Stage. Since the outbreak of COVID-19, the prediction of confirmed cases has become a hot topic. Accurate prediction of confirmed cases can help the government to arrange appropriate preventive measures and allocate medical resources scientifically (For the same reason, forecasting the trend of other epidemics accurately is also important. This method can be used to predict the confirmed cases of other epidemic diseases.). The increase of cumulative confirmed cases just meets the requirements of the FGM and buffer operator. To be specific, in the early stage, the number of confirmed cases is increasing rapidly, and there is the possibility of outbreak at any time. Therefore, this study uses the strengthening buffer operator to simulate this increasing mechanism. In the empirical analysis, we choose the data of Italy, Spain, the United Kingdom, Japan, the United States, and the Republic of South Africa to demonstrate improved prediction accuracy. The data of cumulative confirmed cases 
TABLE 1: The buffer levels of the strengthening buffer operator for different countries.

\begin{tabular}{cccccccc}
\hline & ITA & ESP & GBR & JPN & USA & RSA & CHN \\
\hline SBO & 0.25 & 0.41 & 0.43 & 0.1 & 0.2 & 0.62 & 0 \\
\hline
\end{tabular}

ITA, EPS, GBR, JPN, USA, RSA, and CHN indicate Italy, Spain, the United Kingdom, Japan, the United States, the Republic of South Africa, and mainland China separately.

TABLE 2: The prediction performance.

\begin{tabular}{|c|c|c|c|c|c|c|}
\hline & \multicolumn{2}{|c|}{ FGM } & \multicolumn{2}{|c|}{$\mathrm{FGM}+\mathrm{SBO}$} & \multicolumn{2}{|c|}{ GM $(1,1)$} \\
\hline & MAPE (\%) & RMSE & MAPE (\%) & RMSE & MAPE (\%) & RMSE \\
\hline ITA & 11.981 & 1205.576 & 3.494 & 271.156 & 25.601 & 1845.407 \\
\hline ESP & 40.725 & 2543.256 & 15.663 & 837.511 & 55.579 & 2940.273 \\
\hline GBR & 23.465 & 370.812 & 11.868 & 168.288 & 30.117 & 377.849 \\
\hline JPN & 2.339 & 7.204 & 2.353 & 6.804 & 9.244 & 23.669 \\
\hline USA & 38.152 & 1806.701 & 31.574 & 1503.262 & 52.140 & 2229.252 \\
\hline RSA & 46.091 & 497.059 & 29.366 & 303.475 & 48.347 & 491.166 \\
\hline $\mathrm{CHN}$ & 7.179 & 611.061 & 7.179 & 611.061 & 26.316 & 2267.579 \\
\hline
\end{tabular}

ITA, EPS, GBR, JPN, USA, RSA, and CHN indicate Italy, Spain, the United Kingdom, Japan, the United States, the Republic of South Africa, and mainland China separately.
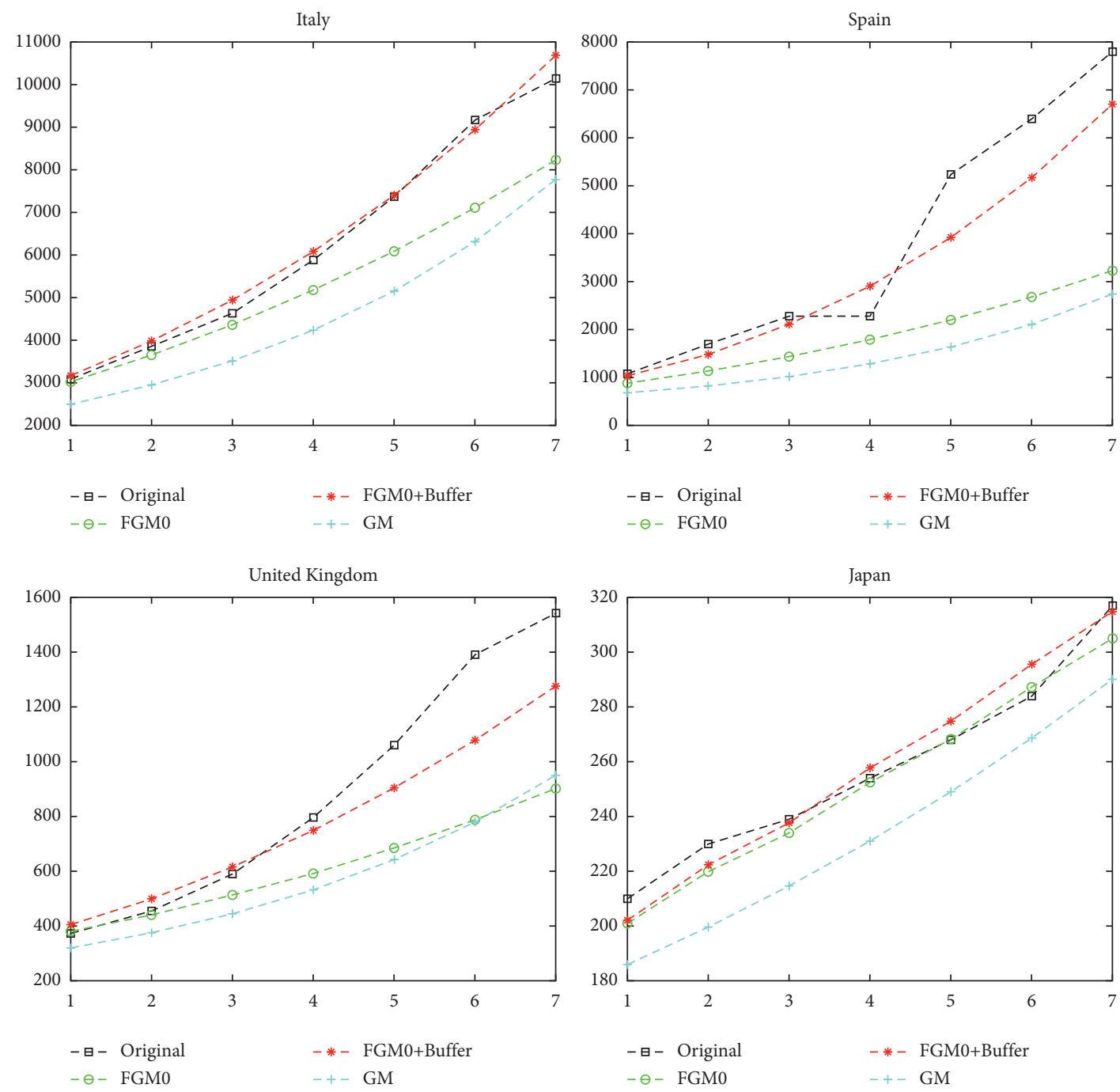

FIGURE 2: The prediction performance in the early stage (part 1). 

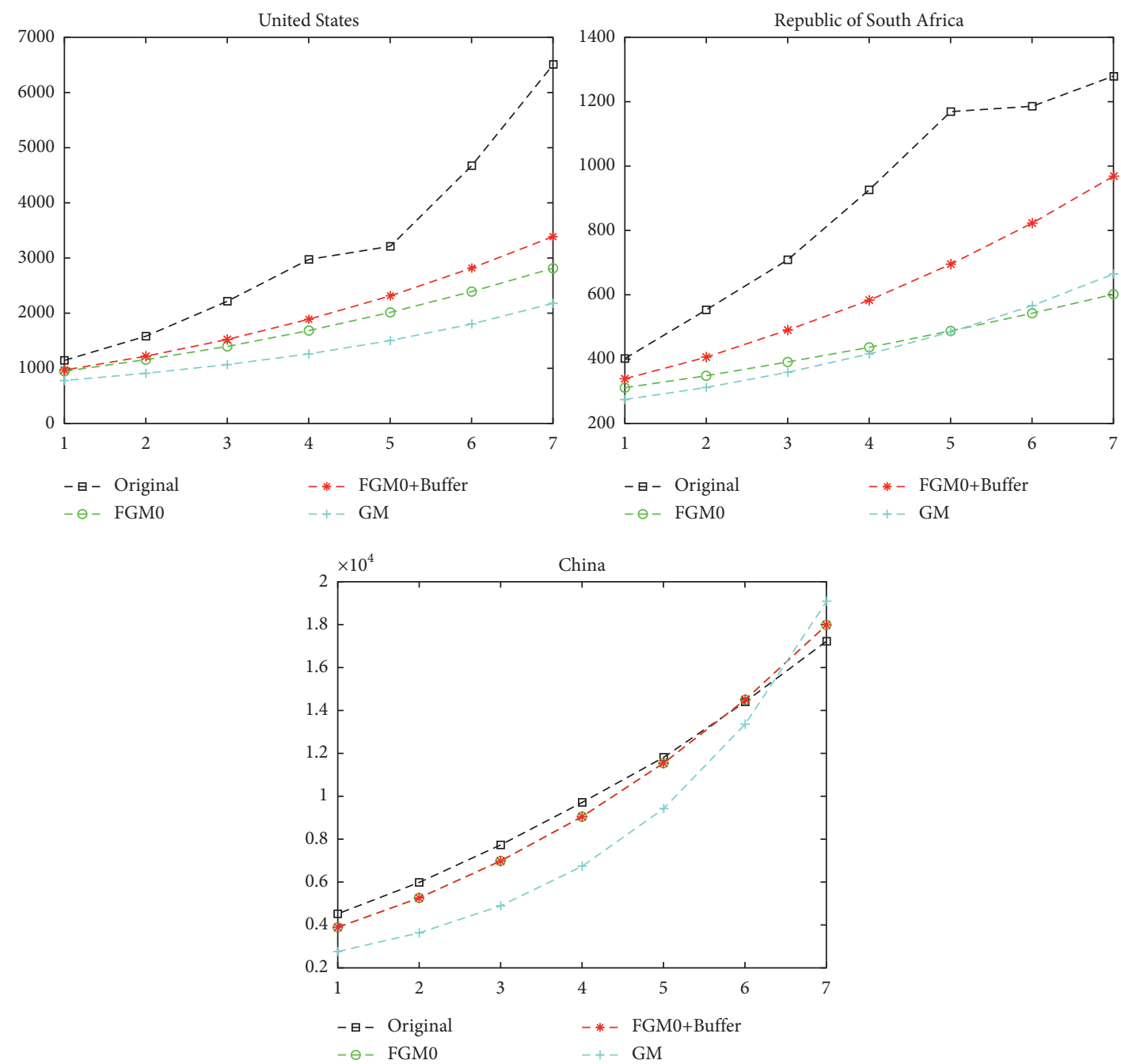

Figure 3: The prediction performance in the early stage (part 2).

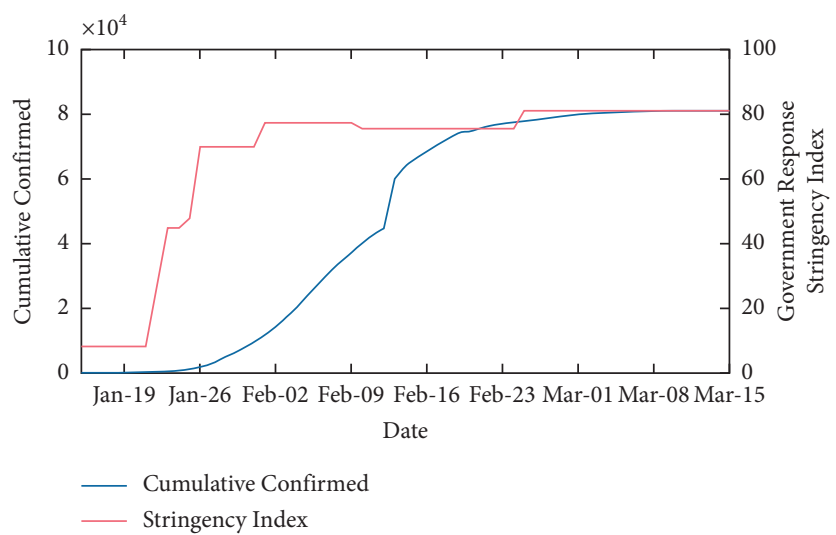

Figure 4: China cumulative confirmed cases and stringency index (January 15, 2020-March 15, 2020).

are collected from the Center for Systems Science and Engineering (CSSE) at Johns Hopkins University. This study chooses the first 12 days when the cumulative confirmed cases increase continuously as the training set to achieve the orders of the strengthening buffer operator. The training set can be divided into two parts.

The first part only includes the first five observations, the minimum requirement for GM modeling. The other part, 


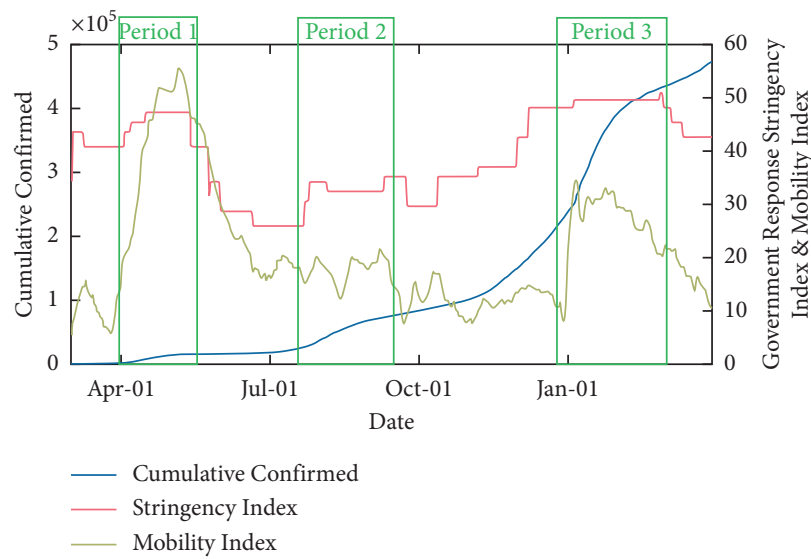

FIgURE 5: Japan cumulative confirmed cases, stringency index, and mobility index (March 1, 2020-March 31, 2020).

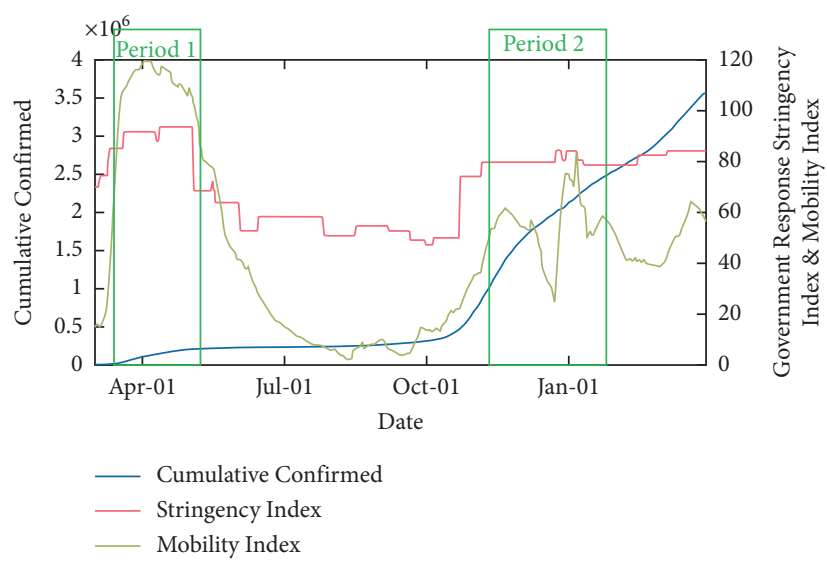

FIGURE 6: Italy cumulative confirmed cases, stringency index, and mobility index (March 1, 2020-March 31, 2020).

which includes other seven observations, is used to compare the prediction accuracy so that the buffer level (or fractional order) can be determined. The buffer levels of the strengthening buffer operator for the sample countries are shown in Table 1. With the data buffered by the strengthening buffer operator, this paper predicts with the FGM. Every time the model predicts a new value, we take the new value as the given value and achieve a new order for the FGM. Consequently, the order of the FGM is not fixed.

Then, this paper selects the last five data in the training set as the in-sample data to predict the next seven days' cumulative confirmed cases. The benchmark models include the FGM without the strengthening buffer operator and GM $(1,1)$. Both of them are suitable for the prediction based on small sample data. The criteria include mean absolute percentage error (MAPE) and root mean square error (RMSE), two most widely used criteria in prediction performance comparison. The results are shown in Table 2.

The results in Table 2 show that the FGM achieves smaller MAPE and RMSE than GM $(1,1)$ for all seven countries. FGM with the strengthening buffer operator always performs better than the FGM in RMSE. FGM with the strengthening buffer operator also achieves smaller MAPE when it predicts the cumulative confirmed cases of Italy,
Spain, the United Kingdom, the United States, and the Republic of South Africa. Figures 2 and 3 indicate the prediction results provided by the FGM with the strengthening buffer operator which are more consistent with the true values. As a result, in the early stage of the epidemic, the prediction accuracy can be improved by considering the strengthening buffer operator and modeling with the FGM. The practitioners can continuously add new data and use this method to predict the future cumulative confirmed cases continually when the scientists and doctors do not find the effective way to prevent the epidemic.

\subsection{The Prediction of COVID-19 Confirmed Cases after the} Prevention Measures Work. After the outbreak of COVID19 for a period of time, the public knows and adopts the prevention method. Consequently, the growth of the confirmed cases is slowing down. As a result, the weakening buffer operator should be utilized to predict the cumulative confirmed cases after the governments issue prevention measures, and the public respond positively to them. The severity of the governments' prevention and control measures can be evaluated by the Oxford COVID-19 Government Response Tracker Stringency Index which is collected 

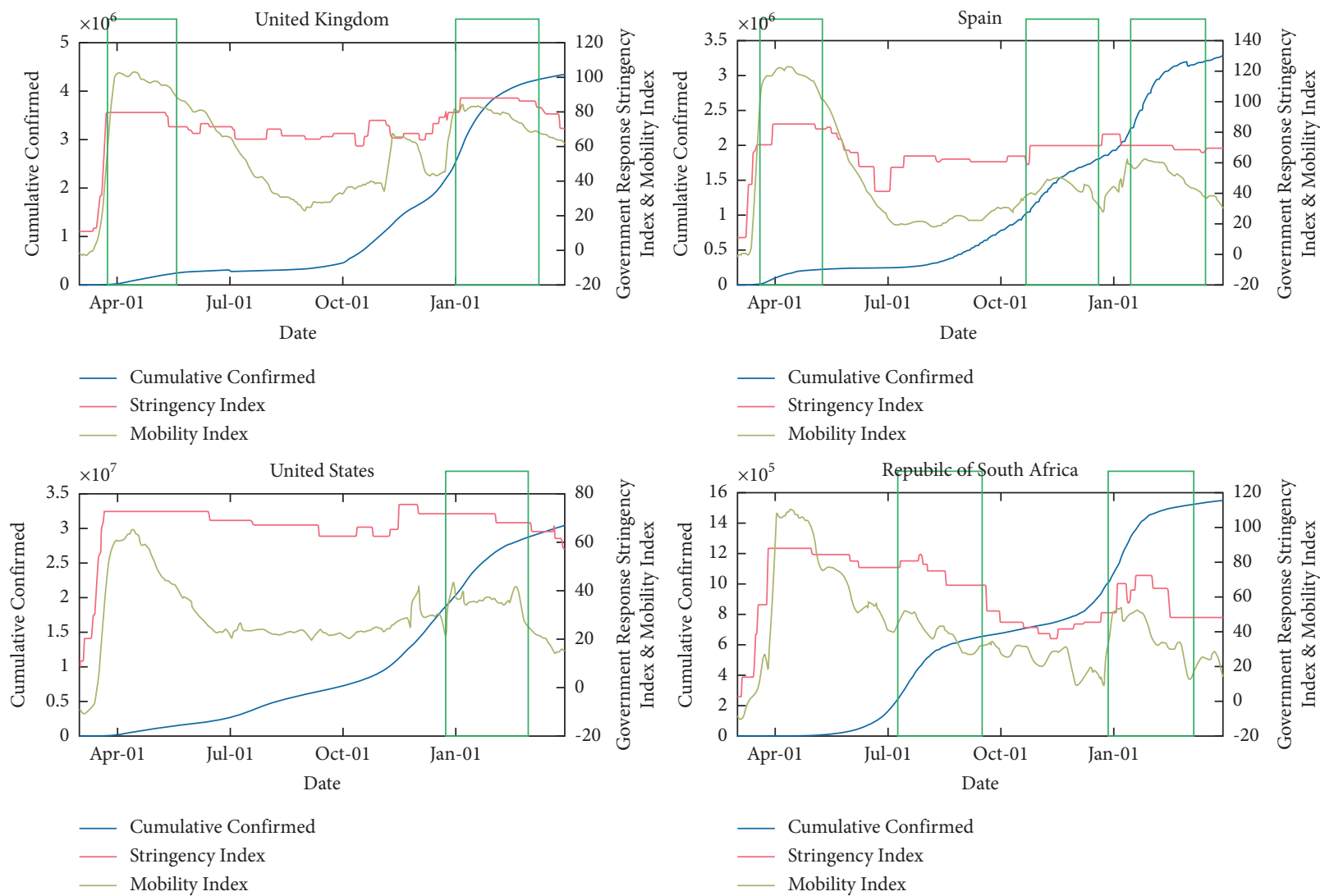

FIgURE 7: Cumulative confirmed cases, stringency index, and mobility index in the United Kingdom, Spain, the United States, and the Republic of South Africa (March 1, 2020-March 31, 2020).

TABLE 3: The buffer levels of the weakening buffer operator for different countries.

\begin{tabular}{cccccccc}
\hline & ITA & ESP & GBR & JPN & USA & RSA & CHN \\
\hline SBO & 0.08 & 0.17 & 0 & 0.11 & 0.01 & 0.15 & 1.09 \\
\hline
\end{tabular}

ITA, EPS, GBR, JPN, USA, RSA, and CHN indicate Italy, Spain, the United Kingdom, Japan, the United States, the Republic of South Africa, and mainland China separately.

TABLE 4: The prediction performance.

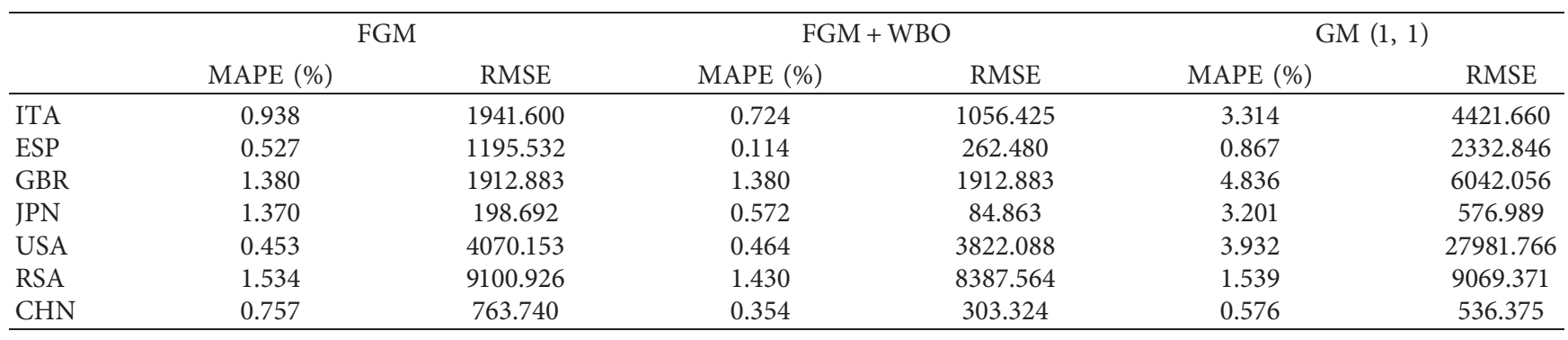

ITA, EPS, GBR, JPN, USA, RSA, and CHN indicate Italy, Spain, the United Kingdom, Japan, the United States, the Republic of South Africa, and mainland China separately.

from publicly available sources such as news articles and government press releases. COVID-19 Community Mobility Reports published by Google are considered to measure the effect of each government's control measures. The Community Mobility Report dataset is presented by location and highlights the percent change in visits to places such as retail and recreation places, grocery and pharmacy stores, parks within a geographic area, and staying at residence. Consider grocery and pharmacy stores are very necessary in daily life, and the visits to parks also depend on the weather, for example, the public go to parks more often in summer than in winter. Moreover, the most important prevention 

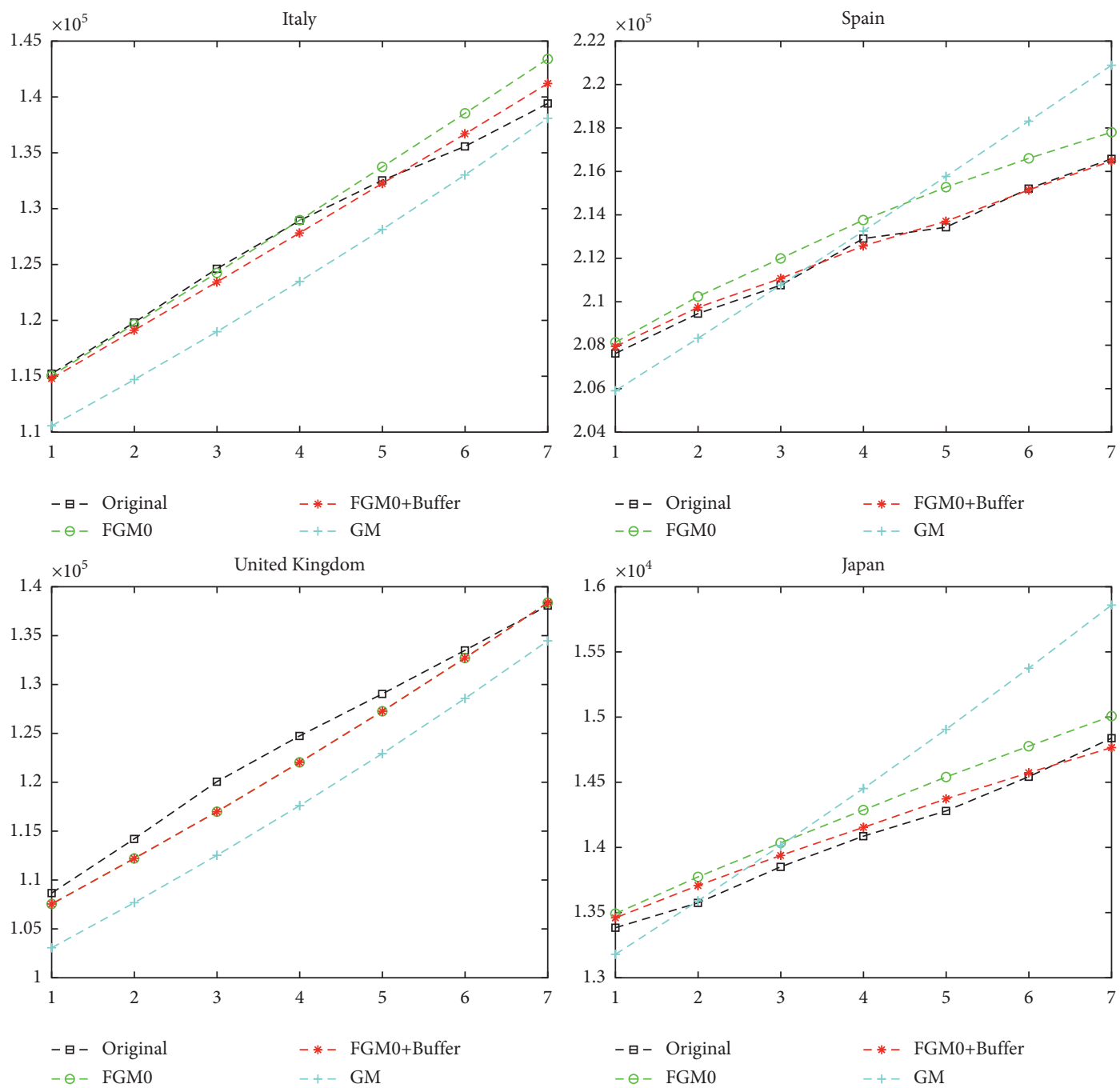

FIGURE 8: The prediction performance after the government's control measures took effect (part 1).

measure is home quarantine order. This paper only considers the percent change in visits to places such as retail and recreation and staying at residence. Due to the home quarantine order, people reduce unnecessary visits. The percentage change in visits to places such as retail and recreation is negative, and the percentage change in staying at home is positive. This paper considers the absolute percentage change. Because people visit retail and recreation places more frequently in the weekend than in the workdays, the seven-day moving average is also applied in processing the data. This paper uses the seven-day moving average of the summation of the absolute percentage changes in visits to retail and recreation places and staying at home to measure the level that the public comply with the government's prevention measures.

Mainland China was the first region which controlled the spread of COVID-19 well because the Chinese government took strong prevention measures, and the public actively cooperated. Figure 4 shows the stringency index and the cumulative confirmed cases in China from January 15, 2020, to March 15, 2020 (Because Google has no business in mainland China, Community Mobility Reports do not contain any data about mainland China.). It can be found that, about twenty days (from January 26 to February 16) after the stringency index increased dramatically, the daily confirmed cases began to decrease (There was a sharp increase on February 12 because the clinically diagnosed cases were included into the confirmed cases. The detection ability of nucleic acid in the early stage is limited, and there are errors in nucleic acid detection. This measure enabled patients to receive intensive treatment earlier while reducing the risk of community transmission.). Figure 5 illustrates the cumulative confirmed cases, stringency index, and mobility index of Japan. It can be found that there are three periods in that the growth rate of cumulative confirmed cases slowed down. Period 1 is in the early spring, and period 3 is in the winter. Both seasons are easy to break out for COVID-19. However, due to the strict prevention of the government (measured by the stringency index) and the active cooperation of people (measured by the mobility index), the number of confirmed cases has changed from rapid growth to low growth. Part of the reasons for the slow growth in period 2 is summer is not the high-incidence season. Refer to Italy; there are two periods in that the growth rate of 

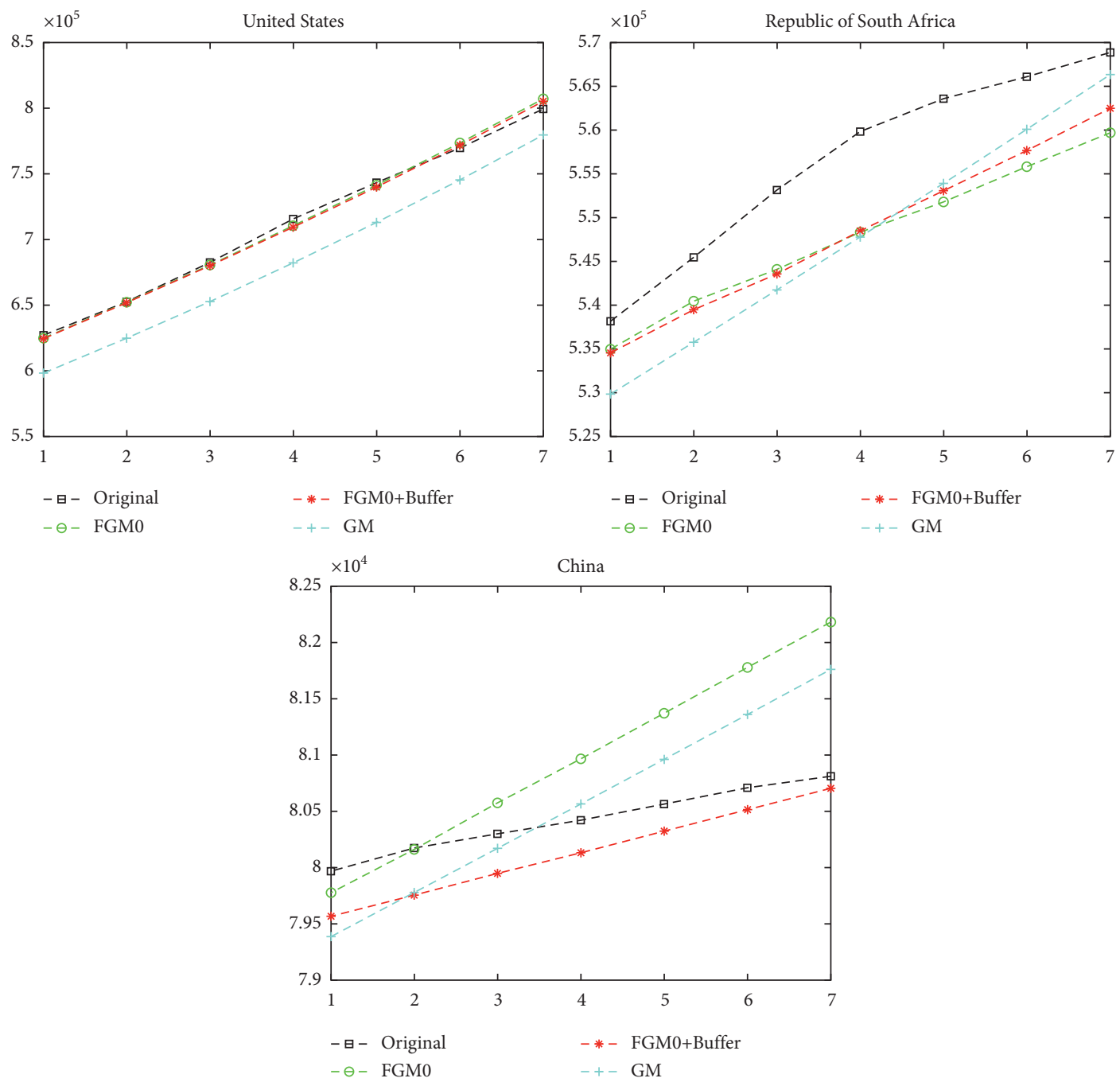

FIGURE 9: The prediction performance after the government's control measures took effect (part 2).

TABLE 5: The prediction performance in the second outbreak.

\begin{tabular}{|c|c|c|c|c|c|c|}
\hline & \multicolumn{2}{|c|}{ FGM } & \multicolumn{2}{|c|}{$\mathrm{FGM}+\mathrm{WBO}$} & \multicolumn{2}{|c|}{ GM $(1,1)$} \\
\hline & MAPE (\%) & RMSE & MAPE (\%) & RMSE & MAPE (\%) & RMSE \\
\hline ITA & 2.166 & 29078.137 & 1.314 & 16297.286 & 4.490 & 43236.182 \\
\hline ESP & 1.153 & 15756.608 & 1.246 & 16753.955 & 1.307 & 15307.242 \\
\hline GBR & 0.285 & 4627.756 & 0.285 & 4627.756 & 1.697 & 20850.792 \\
\hline JPN & 0.788 & 3496.378 & 0.716 & 2499.642 & 2.190 & 7388.862 \\
\hline USA & 1.045 & 185861.267 & 1.012 & 180634.907 & 1.925 & 290226.135 \\
\hline RSA & 0.488 & 8024.826 & 0.457 & 6444.510 & 2.375 & 29474.302 \\
\hline
\end{tabular}

(1) ITA, EPS, GBR, JPN, USA, and RSA indicate Italy, Spain, the United Kingdom, Japan, the United States, and the Republic of South Africa separately. (2) Since there is no large-scale outbreak in China at the end of 2020, this paper did not analyze China's data here.

cumulative confirmed cases decreased (shown in Figure 6). In both periods, the stringency index and the mobility index increased. Especially in the first half of the second period, there was an obvious turning point in the growth of the cumulative confirmed cases. However, due to the impact of Christmas and New Year holidays, the mobility index dropped sharply, which did not further reduce the growth rate of confirmed cases. From the growth chart of confirmed cases in China, Japan, and Italy, we found a similar rule: the government's strict prevention and control measures and the public's cooperation (such as reducing aggregation and home isolation) can slow down the growth rate of confirmed cases. The growths of confirmed cases in the United Kingdom, Spain, the United States, and the Republic of South Africa also show this rule (see Figure 7). 

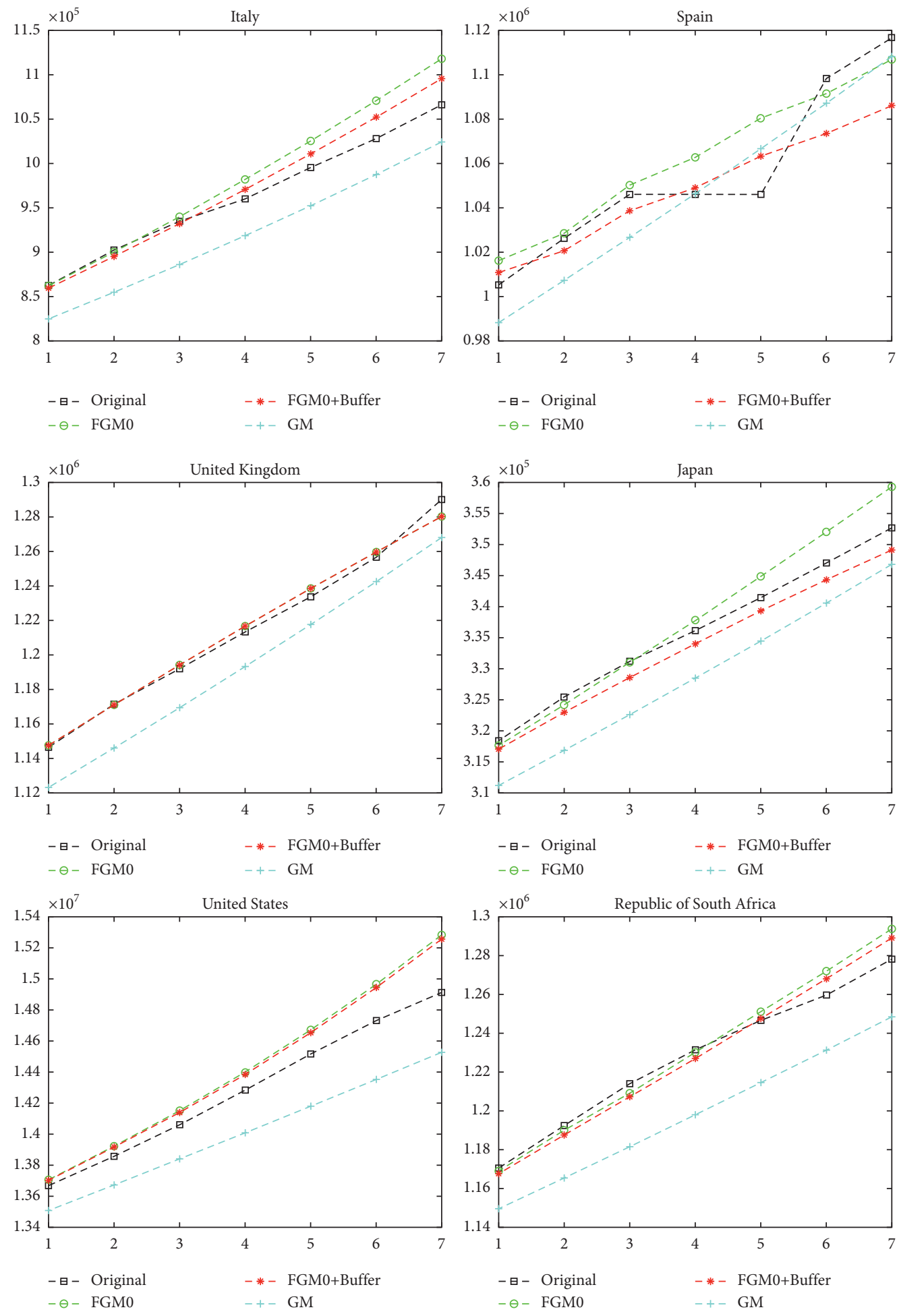

FIGURE 10: The prediction performance after the government's control measures took effect in the second outbreak of COVID-19.

Because the government's strict prevention and control and the public's cooperation can slow down the growth rate of confirmed cases, it is necessary to bring in the weakening buffer operator. Since the maximum incubation period of COVID-19 is 14 days proposed by both the WHO and European Center for Disease Prevention and Control [38], the growth rate of cumulative confirmed cases slowed down from the fourteenth day after the stringency index and mobility index increased. With the same method of estimating the strengthening buffer operator, the starting point of the training set is the 10th day after the stringency index and mobility index increased in the first outbreak (for the Northern Hemisphere countries, the first outbreak started from March). The orders of weakening buffer operators for 
the sample countries are shown in Table 3. Because the buffer operator is used together with the FGM and FGM is suitable for the prediction of nonexponential growth, the order of the weakening buffer operator is zero for the United Kingdom.

Then, this paper used the last five data of the training set as the in-sample data to predict the next seven data. The results indicate that the FGM with the weakening buffer operator has the smallest RMSE for all the countries. It also has the smallest MAPE for Italy, Spain, the United Kingdom, Japan, the Republic of South Africa, and China, except the United States (Table 4). The prediction results are also displayed in Figures 8 and 9.

In the end of 2020, COVID-19 broke out twice in a lot of countries. When the governments realized the second outbreak of the epidemic, they raised the level of government prevention and control again. At the same time, residents reduced unnecessary travel and increased the time of home isolation. So, this paper used the same fractional order of the weakening buffer operator and the first five data after the government stringency index and mobility index both increased for ten days to test the reliability. The results are indicated in Table 5. It can be found that, except Spain, the FGM with the fractional weakening buffer operator performs better than other two models. Although the FGM with the fractional weakening buffer operator does not achieve the smallest MAPE and RMSE, it beats GM $(1,1)$ with Spain's data, and it has similar results to the FGM (shown in Figure 10). Also, the FGM with the fractional weakening buffer operator and the FGM have similar prediction accuracy.

Some of the characteristics of COVID-19 have been mastered by scientists; however, because of its strong variability and high contagion, COVID-19 is still not defeated by human beings. With the improvement of vaccination rate and public awareness of self-protection, the number of newly diagnosed patients in most countries is no longer increasing exponentially. The practitioners can use the FGM with the weakening buffer operator to predict the trend of the epidemic through continuously adding new data to estimate the appropriate buffer levels. Because the FGM is suitable for predicting nonexponential growth $[18,39]$ and the weakening buffer operator reflects the effect of government prevention measures and vaccination, the combination of the FGM and weakening buffer operator through the rolling mechanism is suitable in the future. However, when a more infectious mutation appears, the strengthening buffer operator should be considered.

\section{Conclusion}

In this study, we use the FGM combined with the fractionalorder buffer operator to predict the cumulative confirmed cases of COVID-19. FGM is one of the forefront methods in grey system modeling. It makes full use of known information under the condition of less data environment and has an excellent performance in predicting the future trend. Combining the fractional-order buffer operator with the FGM in an appropriate way can improve the forecasting accuracy. By analyzing the characteristics of the epidemic in different stages, this study proposes to introduce different buffer operators in the prediction process. The examples of Italy, Spain, the United Kingdom, Japan, the USA, the Republic of South Africa, and China show that the FGM combined with the fractional-order buffer operator can improve the prediction accuracy.

In practice, this paper finds that only when government pandemic control measures are in place and people respond actively to these measures, the spread of COVID-19 is deterred. Appropriate use of the buffer level according to government stringency and mobility can improve the predictive accuracy. Buffer levels are obtained from countries' historical data.

\section{Data Availability}

The data on cumulative confirmed cases are collected from the Center for Systems Science and Engineering (CSSE) at Johns Hopkins University (https://coronavirus.jhu.edu/ map.html). The data on the stringency index are provided by the Oxford COVID-19 Government Response Tracker (http://www.bsg.ox.ac.uk/covidtracker). The data on the mobility index are based on the COVID-19 Community Mobility Reports published by Google. The stringency index and the number of cumulative confirmed cases are available at https://covid19datahub.io/. The COVID-19 Community Mobility Reports are downloaded from https://www.google. com/covid19/mobility/. The data used to support the findings of this study are also available from the corresponding author upon request.

\section{Conflicts of Interest}

The authors declare that there are no conflicts of interest regarding the publication of this article.

\section{Acknowledgments}

This work was supported by the National Natural Science Foundation of China (71701127, 42176217, and 72073086) and National Statistical Science Research (2020LY048).

\section{References}

[1] K. M. Furati, I. O. Sarumi, and A. Q. M. Khaliq, "Fractional model for the spread of covid-19 subject to government intervention and public perception," Applied Mathematical Modelling, vol. 95, no. 89-105, pp. 89-105, 2021.

[2] A. E. Aboanber, A. A. Nahla, and S. M. Aljawazneh, "Fractional two energy groups matrix representation for nuclear reactor dynamics with an external source," Annals of Nuclear Energy, vol. 153, 2021.

[3] C. Xu, Z. Liu, M. Liao, P. Li, Q. Xiao, and S. Yuan, "Fractionalorder bidirectional associate memory (BAM) neural networks with multiple delays: the case of HOPF bifurcation," Mathematics and Computers in Simulation, vol. 182, pp. 471-494, 2021.

[4] P. Li, L. Xiong, Z. Wang, M. Ma, and J. Wang, "Fractionalorder sliding mode control for damping of subsynchronous 
control interaction in DFIG-based wind farms, "Wind Energy, vol. 23, no. 3, pp. 749-762, 2020.

[5] J. L. Deng, "Control problems of grey systems," Systems \& Control Letters, vol. 1, no. 5, pp. 288-294, 1982.

[6] S. Liu and Y. Lin, Grey Information: Theory and Practical Applications, Springer-Verlag, London, UK, 2006.

[7] T. H. M. El-Fouly, E. F. El-Saadany, M. M. A. Salama, F. E. Salama, and M. Ma, "Improved grey predictor rolling models for wind power prediction," IET Generation, Transmission \& Distribution, vol. 1, no. 6, pp. 928-937, 2007.

[8] T.-Y. Pai, "Gray and neural network prediction of effluent from the wastewater treatment plant of industrial park using influent quality," Environmental Engineering Science, vol. 25, no. 5, pp. 757-766, 2008.

[9] R.-C. Tsaur, "Forecasting analysis by using fuzzy grey regression model for solving limited time series data," Soft Computing, vol. 12, no. 11, pp. 1105-1113, 2008.

[10] L. Tu and Y. Chen, "An unequal adjacent grey forecasting air pollution urban model," Applied Mathematical Modelling, vol. 99, pp. 260-275, 2021.

[11] J. Deng, The Basis of Grey Theory, Press of Huazhong University of Science \& Technology, Wuhan, China, 2002.

[12] Z.-X. Wang, Y.-G. Dang, and S.-F. Liu, "Unbiased grey verhulst model and its application," Systems EngineeringTheory \& Practice, vol. 29, no. 10, pp. 138-144, 2009.

[13] C.-I. Chen, "Application of the novel nonlinear grey Bernoulli model for forecasting unemployment rate," Chaos, Solitons \& Fractals, vol. 37, no. 1, pp. 278-287, 2008.

[14] Z.-X. Wang, K. W. Hipel, Q. Wang, and S.-W. He, "An optimized NGBM $(1,1)$ model for forecasting the qualified discharge rate of industrial wastewater in China," Applied Mathematical Modelling, vol. 35, no. 12, pp. 5524-5532, 2011.

[15] L. Wu, S. Liu, L. Yao, S. Yan, and D. Liu, "Grey system model with the fractional order accumulation," Communications in Nonlinear Science and Numerical Simulation, vol. 18, no. 7, pp. 1775-1785, 2013.

[16] F. Liu, W. Guo, R. Li, and J. Liu, "Improved load forecasting model based on two-stage optimization of gray model with fractional order accumulation and Markov chain," Communications in Statistics-Theory and Methods, vol. 50, pp. 1-15, 2019.

[17] L. Zeng, "Analysing the high-tech industry with a multivariable grey forecasting model based on fractional order accumulation," Kybernetes, vol. 48, no. 6, pp. 1158-1174, 2019.

[18] W. Wu, X. Ma, B. Zeng, Y. Wang, and W. Cai, “Application of the novel fractional grey model FAGMO $(1,1, k)$ to predict China's nuclear energy consumption," Energy, vol. 165, no. B, pp. 223-234, 2018.

[19] L. Liu, Y. Chen, and L. Wu, "Forecasting confirmed cases, deaths, and recoveries from covid-19 in China during the early stage," Mathematical Problems in Engineering, vol. 2020, Article ID 1405764, 4 pages, 2020.

[20] S. Liu, "The three axioms of buffer operator and their application," Journal of Grey System, vol. 3, no. 1, pp. 39-48, 1991.

[21] Y. Dang, S. Liu, B. Liu, and X. Tang, "Study on the buffer weakening operator," Chinese Journal of Management Science, vol. 12, no. 2, pp. 109-112, 2004.

[22] Y. Dang, S. Liu, and C. Mi, "Study on characteristics of the strengthening buffer operators," Control and Decision, vol. 22, no. 7, pp. 730-734, 2007.

[23] Z. Wang, Y. Dang, and S. Liu, "Study on buffer operators with variable weights and their effect strength to original sequence," Control and Decision, vol. 24, no. 8, pp. 1218-1222, 2007.

[24] L. Wu, S. Liu, and Y. Yang, "Using the fractional order method to generalize strengthening buffer operator and weakening buffer operator," IEEE/CAA Journal of Automatica Sinica, vol. 5, no. 6, pp. 1074-1078, 2018.

[25] G. Perone, An ARIMA Model to Forecast the Spread and the Final Size of Covid-2019 Epidemic in Italy, HEDG-WP University of York, London, UK, 2020.

[26] S. K. Sharma, S. Bhardwaj, R. Bhardwaj, and M. Alowaidi, "Nonlinear time series analysis of pathogenesis of covid-19 pandemic spread in Saudi Arabia," CMC-Computers Materials \& Continua, vol. 66, no. 1, pp. 805-825, 2021.

[27] O. D. Ilie, R. O. Cojocariu, A. Ciobica, S. I. Timofte, I. Mavroudis, and B. Doroftei, "Forecasting the spreading of covid-19 across nine countries from Europe, Asia, and the American continents using the ARIMA models," Microorganisms, vol. 8, no. 8, 2020.

[28] S. K. Tamang, P. D. Singh, and B. Datta, "Forecasting of covid19 cases based on prediction using artificial neural network curve fitting technique," Global Journal of Environmental Science and Management, vol. 6, pp. 53-64, 2020.

[29] B. B. Hazarika and D. Gupta, "Modelling and forecasting of covid-19 spread using wavelet-coupled random vector functional link networks," Applied Soft Computing, vol. 96, 2020.

[30] V. K. R. Chimmula and L. Zhang, "Time series forecasting of covid-19 transmission in Canada using lstm networks," Chaos, Solitons \& Fractals, vol. 135, 2020.

[31] S. Khajanchi and K. Sarkar, "Forecasting the daily and cumulative number of cases for the covid-19 pandemic in India," Chaos, vol. 30, no. 7, p. 71101, 2020.

[32] I. Cooper, A. Mondal, and C. G. Antonopoulos, "A sir model assumption for the spread of covid-19 in different communities," Chaos, Solitons, and Fractals, vol. 139, Article ID 110057, 2020.

[33] F. G. Santeramo, M. Tappi, and E. Lamonaca, "On the management of covid-19 pandemic in Italy," Health Policy, vol. 125, no. 8, pp. 995-1001, 2021.

[34] Y. Tsori and R. Granek, "Epidemiological model for the inhomogeneous spatial spreading of covid-19 and other diseases," PLoS One, vol. 16, no. 2, 2021.

[35] J. Chen and $\mathrm{Z}$. Wu, "A positive real order weakening buffer operator and its applications in grey prediction model," Applied Soft Computing, vol. 99, 2021.

[36] X. H. Gao and L. Wu, "Using fractional order weakening buffer operator to forecast the main indices of online shopping in China," Grey Systems: Theory and Application, vol. 9, no. 1, pp. 128-140, 2019.

[37] W. Li and X. Han, "Geometrical variable weights buffer GM $(1,1)$ model and its application in forecasting of China's energy consumption," Journal of Applied Mathematics, vol. 2014, Article ID 131432, 6 pages, 2014.

[38] N. Zaki and E. A. Mohamed, "The estimations of the COVID19 incubation period: a scoping reviews of the literature," Journal of Infection and Public Health, vol. 14, no. 5, pp. 638-646, 2021.

[39] M. Gao, S. Mao, X. Yan, and J. Wen, "Estimation of Chinese $\mathrm{CO}_{2}$ emission based on a discrete fractional accumulation grey model," Journal of Grey System, vol. 27, no. 4, pp. 114130, 2015. 